\title{
Productivity Curve and Social Network Analysis in Science Megaproject Management
}

\author{
Phillip M. Bentley \\ European Spallation Source ESS ERIC, Partikelgatan 2, 224 84, Lund, Sweden \\ E-mail: phil.m.bentley@gmail.com
}

\begin{abstract}
Megaprojects aimed at delivering next-generation, multi-billion euro scientific research facilities are complex and high-risk endeavours, requiring expert knowledge spanning a wide spectrum of technical and administrative fields. Such organisations tend to evolve organically, responding to technical and political challenges. They are almost certain to fail to meet expectations on schedule, budget, and deliverables. Whilst there have been illuminating "top-down" phenomenological megaproject studies recently, this article reports a "bottom-up" perspective on the emergence of these issues. Firstly, the staff productivity distribution curves are analysed at a European science megaproject, and a stratified culture is identified with a small, high productivity "clique", and a vast, low-productivity group of "outsiders" operating at only $50 \%$ of their potential. The social network is then analysed, revealing a dense decision-making group that is only tenuously connected to technical expert teams via hierarchy. Staff inefficiency is linked to superfluous roles in middle management, carrying increased bureaucratic burdens and a financial loss $\sim 10 \%$ of the annual salary budget. Corrective suggestions are given, for the current megaproject and future activities to mitigate these causes. This should help to reduce some of the overspend, schedule overrun, and reductions in ambition and scope that have become megaproject norms.
\end{abstract}




\section{Introduction}

This is the third article in a series examining scientific megaprojects. The first paper reported the development of a technical strategy [1] for scientific megaproject instrumentation, where each unit is a bespoke design as part of a larger suite. The second [2] demonstrated the use of metaheuristics in the optimal design of the facility, where the performance metrics are non-linear functions with many parameters. The present article addresses worker productivity and organisational structure in supporting the design and deployment of hardware assemblies in a science megaproject.

The construction of large science facilities falls into a subset of megaprojects. Using Flyvbjerg's definition [3], a megaproject is a project of several years duration; costing in excess of $€ 1$ billion; with multiple public and private stakeholders; and where the project benefits impact on millions of people. As such, it should not be a surprise that these projects fail to achieve the stated ambitions within the agreed budget and schedule.

At high levels, Flybjerg and colleagues ([4] and refs therein) document common megaproject issues. The long duration of these activities tends to lead to continuously changing leadership by planners and managers without deep knowledge or experience in the required technical areas, and unsurprisingly therefore also significant reductions to the scope and ambition of the project.

Decision making processes are hampered by many stakeholders possessing conflicting interests. Whilst it is to be expected that reaching new scientific horizons requires some novelty and non-standard technology, there is also widespread uniqueness bias - in the case of instrumentation sub-projects, for example, uniqueness bias may result in some fraction of project failing to consider standard solutions correctly in a game tree [1] and instead pursue bespoke solutions at considerably greater cost for little gain, or even strive towards a demonstrably inferior result [5].

Science megaprojects are usually funded publicly from regional and/or country sources, with the result that principal-agent problems and rent-seeking behaviour are common, tolerated, and sometimes expected. Planning is optimistic and underestimates or ignores high risk events.

The combination of all of these results in a "break-fix" model: when the optimistic planning reaches the stage that it is unambiguously divergent from reality (as happens $90 \%$ of the time across megaprojects as a whole [4]), the projects undergo some form of replanning or restructuring. This might be a regular process that created the baseline situation in the present article after a large number sub-project reviews every 6-12 months, or it can be a large and dramatic restructuring of the entire organisation and re-baselining of the project as described later.

To offer a different and complementary perspective to the "top-down" macro studies, this article takes a more "bottom-up", micro study perspective on a megaproject. Contributing factors are revealed at the coalface which subsequently blossom into the "iron law" of megaprojects [4] - the inevitable failure to meet budget, schedule and expectations on outcomes. It is not claimed that these core project issues 
are the root cause of the iron law across all megaprojects, and how widespread these phenomena are is a worthy subject of further study.

Specifically, this paper attempts to collect together objective data on the work being done in a science megaproject, and use it to answer pertinent questions:

(i) How much work are employees doing in support of the project?

(ii) Where in the organisational structure are they working?

(iii) What kind of organisational environment do they experience?

(iv) How might one improve their productivity and environment?

It is worth noting that the objective in examining worker productivity is not to identify the lowest productivity staff to be fired - a common mistake with prominent champions (e.g. Welch [6]). Instead, this article will demonstrate how staff productivity curves and the network of social interactions can be used to characterise the working environment objectively. With this information, one can take steps to improve the social fabric as a whole.

By worker productivity, there are two types of deliverable that are quantified as being essential to the organisation under study. The first type of output is in the form of scientific research articles, some of which are catalysed by attempting to construct a next-generation science facility. As the boundaries of technical feasibility are probed, new insights and methods are developed by the scientists and engineers working in the project, and these result in shared knowledge to be disseminated.

The second type is a form of technical administration, a collaborative, online, knowledge-sharing system, within which are shared technical drawings, tables of specifications, procedures, requirements, test results, and outcomes in the support of the organisation's activities of designing and building scientific hardware. For simplicity, it will be called "administration" for the remainder of this article.

A more complete picture could be obtained by mining software version control, engineering collaboration tools, and calendar data, but those systems were out of scope of the current study due to constraints regarding privacy. Nonetheless, the results of software sprints; engineering work; minutes, actions and decisions from meetings are all condensed into articles on the administrative sharing system, usually by those who are most directly involved in the matters concerned. This means that the administration curves offer a measure of that effort by proxy. The data that appear in this article have been anonymised to protect the identities of those concerned. However, they can be easily reconstructed in their entirety from public-facing databases, by anyone with valid login credentials at European academic and technical organisations.

In a modern corporate environment, it is common for employees to undergo regular performance review procedures. Employees are typically categorised into one of a cascade of assessment levels with a normal/bell/Gaussian curve shape:

Outstanding - the employee is exceptional and distinguishes themselves markedly from their peers on almost all assessment criteria 
Above Average - the employee is clearly above average in many areas

Average - the employee performs satisfactorily in most areas of their work

Below Average - a number of areas require structured, agreed improvements to bring the employee up to an acceptable level of performance

Poor - the employee fails to meet expectations in the majority of their work and urgent intervention is required

The application of these categorisations normally attaches set quotas for the organisation in each bracket, to avoid everyone labelling each other as "outstanding". This is known as a Forced Distribution Rating System (FDRS). The famous 20-70-10 rule of J. Welch [6] breaks down the percentage quotas for the high, average and poor performance levels respectively, which is just as arbitrary as a Gaussian curve.

When attached to career progress and pay grades, such assessment scales can have unintended yet rational responses, such as sabotage and personal opportunism at the expense of team progress (see [7] and refs therein), but the major problem is that they simply do not represent reality. Almost 100 years ago, productivity curves were published by Lotka [8] on a subset of scientific researchers. He found that $60 \%$ of them had published a single paper. Furthermore, the fraction of authors publishing $n$ papers is $1 / n^{2}$ : thus, $1 / 4$ of the scientists had 2 papers, $1 / 9$ had 3 papers, and so on. Lotka showed that this behaviour explained publishing across the range of 1-30 papers, and the probability of being at the top end of the scale is $1 / 30^{2} \approx 0.1 \%$. This is an example of a power law distribution. This leads to the question as to how widespread is the power law behaviour, which attracted the interest of Price [9] in the 1960s.

In modern times, one can scrape up-to-date public data sources and compare various individual human endeavours, and arrive at the data shown in figure 1 , indicating that similarities persist today. The $x$-axis is the total human work output $Q$, and the $y$-axis is the probability that a participant will match or exceed $Q$. In the upper left, one sees that all employees match or exceed the work output of the lowest performing individual by definition. Then, as one moves to the right and increases the minimum amount of work done, the curve decreases sharply and mostly in a straight line on a log-log scale over multiple orders of magnitude.

"Books published" are the most prolific authors; "Formula 1 Wins" shows the sum of races won by formula 1 drivers; "Linux source commits" are the top 100 contributors (excluding pull requests) to the Linux kernel, according to GitHub; "LPGA Tour Wins" shows the sum of female golfers' career wins on the LPGA tour; "Patented Inventions" are the most prolific inventors / entrepreneurs by published patent; "PGA Tour Wins" shows the sum of male golfers' career wins on the PGA tour; "Research Papers" (A) and (B) are the same data studied by Lotka and Price [8, 9]; "Tennis Singles Wins" show the sum of individual tennis player wins at international championship level for male (M) and female (W) players; and "Wikipedia edits" show the most prolific Wikipedia editors. 


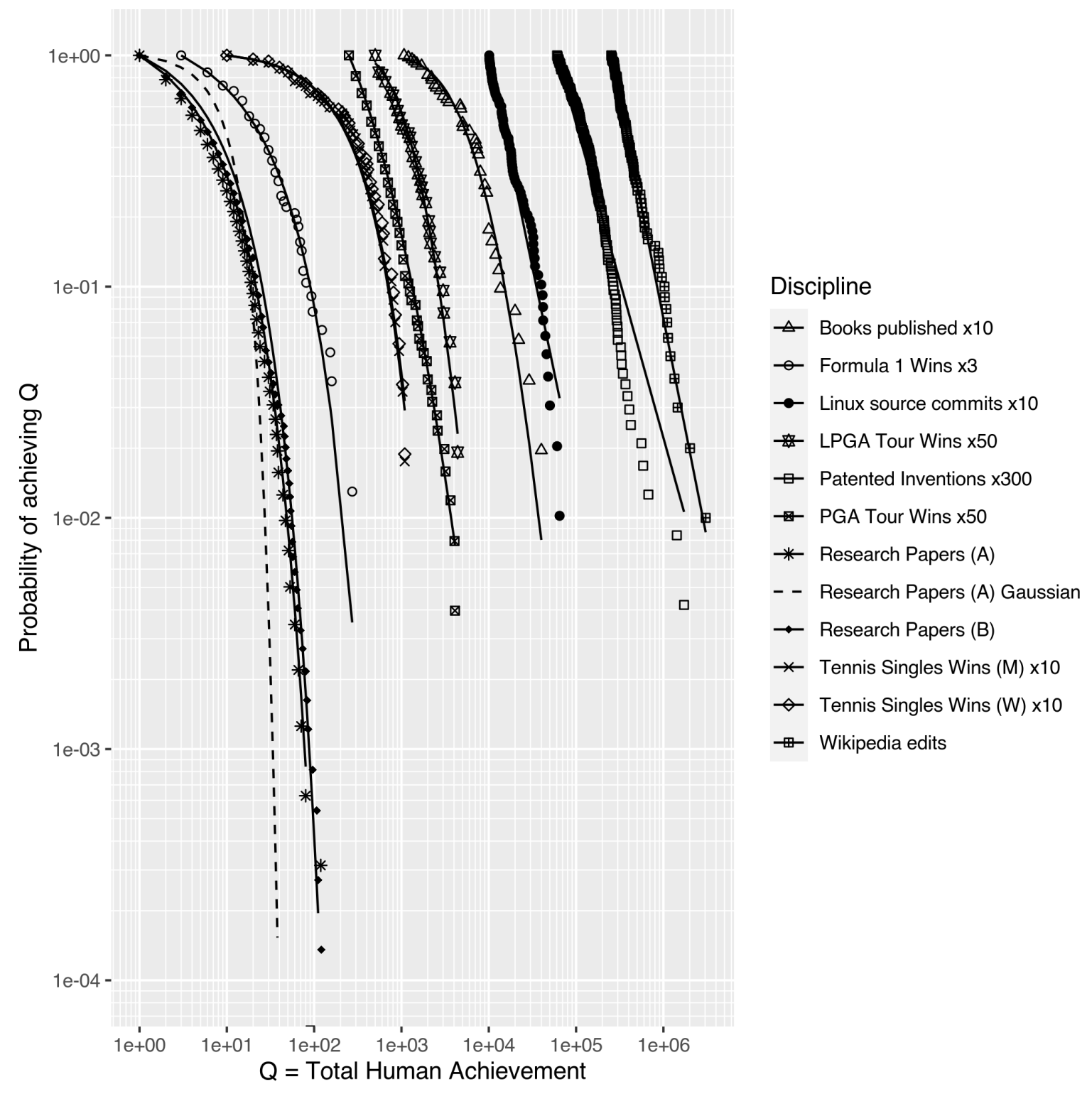

Figure 1. Long tail behaviour in a range of human endeavours. The solid lines are fits to the data as described in the text. The data have been shifted for clarity, as indicated by the multiplicative factors in the legend. The dashed line to the left of the figure shows a normal distribution of productivity. It is common for the bottom-most data points to deviate significantly from the trends, since they have the greatest statistical uncertainties.

The figure reveals quite clearly that none of these are normally-distributed, as shown by the dashed curve for comparison.

The normal curve has a more prominent bulge and curvature at the top (overestimating the output of low productivity people) and a steeper line at the bottom (underestimating the output of high productivity people) than any of the other curve shapes. Thus we see that the assumption of a normal distribution of worker output distorts our perception of employee productivity: 
- In reality, low productivity people do a lot less than one might expect from the gaussian curve (e.g. $1 / 3$ in the figure)

- High performance staff do a lot more than one expects (e.g. between $\times 3$ and $\times 10$ in the figure)

One can immediately see that if one uses a normal distribution to assess employees' prior work and to set forthcoming goals, and negotiates pay based on these assumptions, then one immediately has a deviation from reality in the foundation of the project planning process.

There are more dramatic implications, however. If the performance follows a longtailed distribution, that at least approximates Price's law across a major fraction of the staff, then in a pool of $n$ employees, half of all the work is done by $\sqrt{n}$ staff: e.g. in a company with $n=200$ people there are $\sqrt{200} \approx 14$ employees doing half of all the work. High performance staff are likely to be strong candidates when other opportunities arise. When morale sinks across the organisation, it does not take many resignations before the total productivity is significantly compromised.

A power law is an example of a long-tailed distribution. There are a number of similar, more relevant curve types which will be examined next.

\subsection{Long-Tailed Distributions}

In the curves of figure 1, the probability of equalling or exceeding a work level output is shown - this is the complementary cumulative distribution function (CCDF). It is given the symbol $\bar{F}_{X}(Q)$, and is related to the cumulative distribution function $F_{X}(Q)$ by $\bar{F}_{X}(Q)=1-F_{X}(Q)$, where $F_{X}(Q)$ is the integrated probability $P(X \leq Q)$ that the human output will be less than or equal to $Q$. The functional form of the integral is not important, so long as one remembers that the integration has taken place in order to plot the graphs correctly after describing the probability density, $f(x)$, according to the following sections.

\subsubsection{Power Laws A power law is given by:}

$$
f(x)=\frac{\alpha-1}{x_{\min }}\left(\frac{\alpha}{x_{\min }}\right)^{-\alpha}
$$

The first term is a normalisation constant. $x_{\min }$ is a cut-off below which the law does not apply (because $f(x=0)=\infty$ ). Power laws are exhibited by systems with some degree of scale invariance. For example: the ripples of sand shaped by wind or water are similar in appearance to large dunes of sand in the desert when viewed from the air. The behaviour of sand in fluids is to some degree scale invariant, and the size of the ripples and dunes follow a power law distribution. The same is true for ratios of wealth distribution in human society and population ratios of cities, for example. An overview of the mathematical aspects of power laws is given by Clauset [10].

Power laws usually break down at some limiting boundary: the population of a city cannot expand to infinity due to logistic constraints; the total amount of work done is 
limited by human lifespan. One therefore expects power laws to curve eventually, so some long-tailed distributions with curvature should be considered.

\subsubsection{Gamma Distribution The gamma distribution probability density is:}

$$
f(x ; k, \theta)=\frac{1}{\Gamma(k) \theta^{k}} x^{k-1} e^{-x / \theta}
$$

where $\Gamma(\alpha)$ is the gamma function, $k$ is the "shape" parameter and $\theta$ is the "scale" parameter. A gamma distribution with $k=1$ is an exponential distribution; with $k<1$ the distribution is more steeply increasing towards the origin; and with $k>1$ the distribution exhibits a broad, asymmetric bell-shaped peak at non-zero $x$ values.

What is special about exponential functions, in this context, is the property that the differential of an exponential is an exponential. These functions arise wherever the increase (or decrease) of a quantity is proportional to the size of the quantity. In the workplace, an exponential distribution (or gamma distribution with $k \approx 1$ ) of productivity indicates that workers' opportunities for success in their next activity is in proportion to their previous success record.

This can be easily visualised from the point of view of a manager, who may be more inclined to select an employee for a new task who has a track record, rather than taking a gamble on an unknown employee further down the food chain. Such a scenario produces a $k \approx 1$ gamma distribution, even if all employees put in the same level of effort and possess the same skill level relative to the task difficulty in their area of specialisation. One can easily simulate this, by selecting a small number of agents to be allocated work on each iteration, and the probability of work allocation is by rank-selection in order of the total of prior work done. After a large number of iterations, the integrated scores are distributed according to a gamma distribution with $k \approx 1$. This is illustrated in figure 2. It is unsurprising, therefore, that wealth in industrialised nations has also been found to follow gamma distributions. A good overview of gamma distributions is given by Kleiber and Kotz [11].

For $k<1$, the gamma distribution resembles a curved power law, with a smaller curvature than that of a Gaussian / normal distribution. A competitive social hierarchy model based on wealth exchange reproduces this behaviour, though instead of wealth one might think of "prestige". This is essentially tournament selection. One can model this as a multiple round process, where in each round two individuals are randomly chosen and placed in competition with each other. The individual with the greatest prestige has a probability $q$ of winning the encounter (where $1>q>1 / 2$ ). The loser in the encounter sacrifices a fraction of their prestige $w$ (where $1>w>0$ ) which is transferred to the winner, who takes the full credit for the work activity. After a large number of rounds of this simulation, one obtains a gamma distribution of prestige or credited work, where the shape parameter $k$ of the distribution (equation 2) is given by $k=(1-w) / w$. A simulated example of $k<1$ behaviour is also shown in figure 2 alongside the $k=1$. 


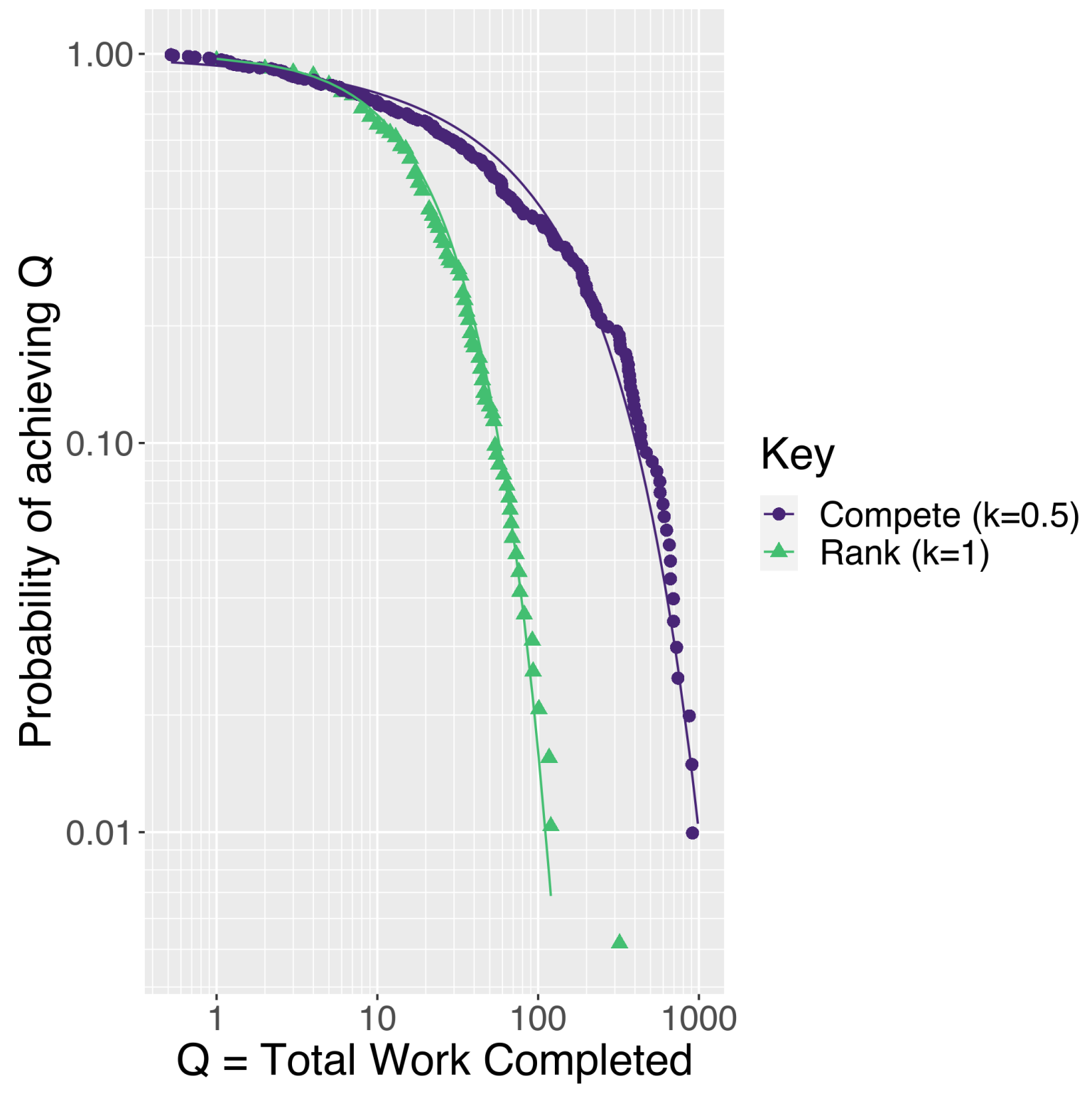

Figure 2. Simulation of two different work environments. Selecting "good" employees for new tasks, with a probability proportional to their previous total work success, generates a gamma distribution with $k \approx 1$ of employee work output across a wide range of the workforce. Having workers mainly competing against each other for reputation gain produces a gamma distribution with $k<1$. The lower "Rank" point is misplaced due to a sampling error, the actual probability is much lower than the $1 / n$ as implied by simulating $n$ entities. 
A $k<1$ gamma distribution would therefore indicate a competitive, and not collaborative, environment. Some organisations actively encourage rivalry of this kind, by assigning $n$ multiple staff members, or teams, to compete at solving the same problem in parallel, with the intention of selecting the best solution from the $n$ options at the end. It is not obvious that the final, individual solution is worth the multiplication of costs $\times n$, and the unintended negative impact can be significant, including unfair and unethical behaviour from some of those who are forced to compete [12].

1.1.3. Log-Normal Distribution The next distribution one should look at is a log normal distribution, with a probability density given by:

$$
f(x ; \mu, \sigma)=\frac{1}{x \sigma \sqrt{2 \pi}} \exp \left(-\frac{(\ln (x)-\mu)^{2}}{2 \sigma^{2}}\right)
$$

This is a normal / Gaussian / bell curve distribution in a logarithmic space. $\mu$ is the mean of the logarithm of the distribution, and $\sigma^{2}$ is the standard deviation of the logarithm of the distribution. It resembles a power law tail with a curve, but the curvature is less than that of a gamma distribution.

One can visualise the origin of this distribution, in the current context, as being related to the central limit theorem (CLT). If one takes a large number of random variables, which are distributed arbitrarily in similar ways, and averages them in a normalised sum, then the resulting distribution will closely approximate a normal distribution. This distribution appears often in physics, and it is known in that community as a Gaussian distribution after the famous physicist, Gauss.

The log normal distribution arises when one takes a large number of random variables, of arbitrary but similar distribution, and takes the geometric mean by multiplying and normalising them. One therefore might expect to see a log normal distribution of outcomes if success depends on chain of conditional probabilities across independent events, described by a large number of variables.

A log normal distribution may emerge in the productivity distribution of work units when a worker's job requires the sequential application of many different skills. This is easy to visualise in the context of golf — as Jack Nicklaus, one of the greats, once said, "The greatest and toughest art in golf is 'playing badly well.' ". What Nicklaus meant, and what has been paraphrased multiple times by others, is that to be competitive one must score well when struggling with poor play, i.e. minimising the impact of mistakes over many holes. Thus, the probability of winning a tournament is the product of a sequence of a large number of random variables governing the players' skills and encountered situations on the course. This is perhaps one contributing reason the golf tours are increasingly interested in compiling detailed player statistics.

One can visualise that an employee with low productivity in a log normal field of work might not actually be a "poor" employee. Figure 3 shows a simulation of four hundred employees, of which two extremely skilled experts were created each having a two specialisations with a 100\% success rate. The remainder of the 7 skills were randomly, uniformly distributed between a success rate of $50-100 \%$ for each of the 7 
Table 1. Characterisation of different working environments from figure 1 based on the productivity curves.

\begin{tabular}{l|r|r}
\hline Discipline & Curve Shape & Implied Classification \\
\hline Authoring books & log-normal & $\mathrm{A}$ \\
Formula 1 & $\Gamma, k \sim 0.4$ & $\mathrm{~B}_{1}$ \\
Linux development & log-normal & $\mathrm{A}$ \\
Female Golf & log-normal & $\mathrm{A}$ \\
Inventing & log-normal & $\mathrm{A}$ \\
Male Golf & log-normal & $\mathrm{A}$ \\
Scientific research & $\Gamma, k=0.4-0.6$ & $\mathrm{~B}_{1}$ \\
Male Tennis & $\Gamma, k \sim 1$ & $\mathrm{~B}_{2}$ \\
Female Tennis & $\Gamma, k \sim 1$ & $\mathrm{~B}_{2}$ \\
Contributing to Wikipedia & power-law & $\mathrm{C}$ \\
\hline
\end{tabular}

tasks that were performed in sequence for each job unit. The success rate is compared with the relative difficulty of the task, again randomly and uniformly generated from $0-100 \%$. Failure at an individual task results in failure for the entire 7 -task job unit. The employees were all trialled against 4000 job units to generate the curve. This should satisfy the requirements for central limit theorem in logarithmic space.

In this simplified example, the skill competences are assumed to be fixed, rather than improving with practice. Despite the presence of stellar abilities in a few employees, the highest cumulative long term performance is recorded by the "jack of all trades" employees, who have acceptable skills in all areas. The resulting curve is very close to a log-normal shape, as expected. The extreme skill level of the two employees is masked by the other challenges, and these still report "poor" performance in this model if they are forced to perform work that draws on several skill sets lying outside their competence area. In the real world, such a scenario is obviously an example of poor management rather than poor employee performance. Replacement of "poor" staff in such a complex, multi-skill environment would be challenging, time consuming, and not guaranteed to improve the overall situation - it would probably be cheaper and easier to re-think the way that work is allocated and distributed. Such an environment probably stands to benefit the most from self organising teams and agile approaches to the management of work (e.g. [13]).

1.1.4. Curve Type Overview Returning to the data of figure 1, the type of curve exhibited by each endeavour, along with the implied environment, is summarised in table 1.

"Type-A" is a log-normal distributed environment, associated with multiple, different skills being applied sequentially in a chain of independent events. "Type$\mathrm{B}$ " is a gamma distributed environment, with people competing against each other for 


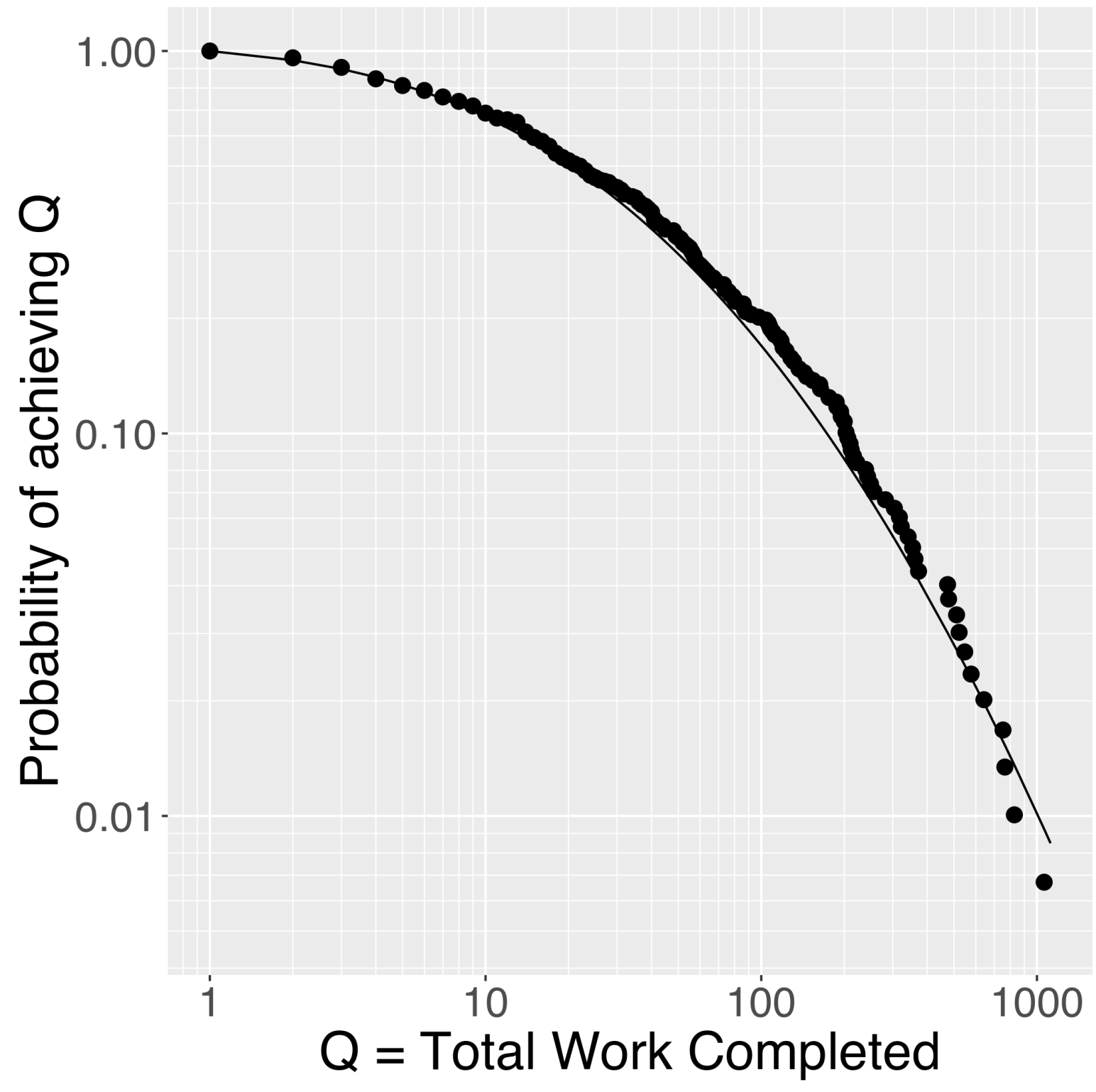

Figure 3. Simulated workforce where successful completion of a job draws on the sequential application of multiple skills. This work environment approximates a lognormal distribution in worker productivity.

future success $\left(\mathrm{B}_{1}\right)$, or where future success is strongly linked with historical success $\left(\mathrm{B}_{2}\right)$. "Type-C" is where only the most prolific contributors have been sampled, and it represents the universal, power-law behaviour at maximum possible worker output.

It is interesting that science appears to be competitive, and that tennis follows the "rich get richer" pattern. One would expect the reverse, since tennis matches are a zero sum competitive game and in science previous work is used to catalyse new ideas and future research work. The other disciplines are all consistent with sequential, multi-skill work, with the exception of wikipedia edits which follow the power law. 


\subsection{Social Network and Centrality}

After quantifying the productivity curves of the workforce, this paper covers the social structure underlying those trends. For this reason, it is appropriate to briefly cover the important mathematics used in such analyses.

The study of social networks is becoming increasingly important in contemporary society, and there are various good, free software tools that allow the user to identify the most important people based on many different models. Mathematically, this falls into the topic of graph theory, the applications of which span criminology, marketing, finance, magnetism, computing, documentation science, and internet search, to name just a few examples.

If one considers a network of social actors, where some actors are socially connected to each other, one can represent this system mathematically as a graph of a set of points or vertices, where the social links between actors are known as edges and frequently drawn as lines. Adjacent vertices are those which are directly connected. A fabricated social network is represented as an example in figure 4.

One might then wish to analyse the graph to determine which of the vertices and edges are the most important, in order to manage the social system optimally. There are many metrics of graph "centrality" that attempt to determine the most important vertices. The simplest measure of centrality is degree centrality, which identifies the vertices with the greatest number of edges. People with a large number of connections are considered important, and people with few connections are considered less so. The raw number of subscribers, or friends, or connections is the main metric used to monitor the relative prestige of participants on many social network platforms. It is also applicable in the spread of diseases, ideas or memes.

Degree centrality is easily calculated, but over-simplistic for many applications. A person with a large number of connections is not necessarily important in the flow of information, since they may be bypassed, or located in a dense network that is not a critical link between peripheral nodes. In figure 4, vertices 1 and 5 have the greatest degree centrality, scoring 5 each. Vertex 1 seems to hold a more prestigious standing in the middle of an important cluster, and is less isolated than vertex 5 . On the other hand, vertex 5 is depended upon by a greater number of vertices and - along with vertex 2 is the sole means of communication between the two halves of the network. Moreover, whilst vertex 1 is embedded in a larger cluster, it can be bypassed two different ways.

Which of these subjective assessments is more important depends on the assumptions about the nature and function of the network. In a corporate setting, do the actors collaborate openly, or are they in competition with each other? Do they freely share information, or do they withhold and/or manipulate the information to gain advantage? It seems important to quantify both scenarios, assuming both are possibilities, in order to minimise organisational risk. One can therefore consider a wider range of centrality measures to capture these other effects.

Betweenness centrality is an attempt to inject some of the information flow into a 


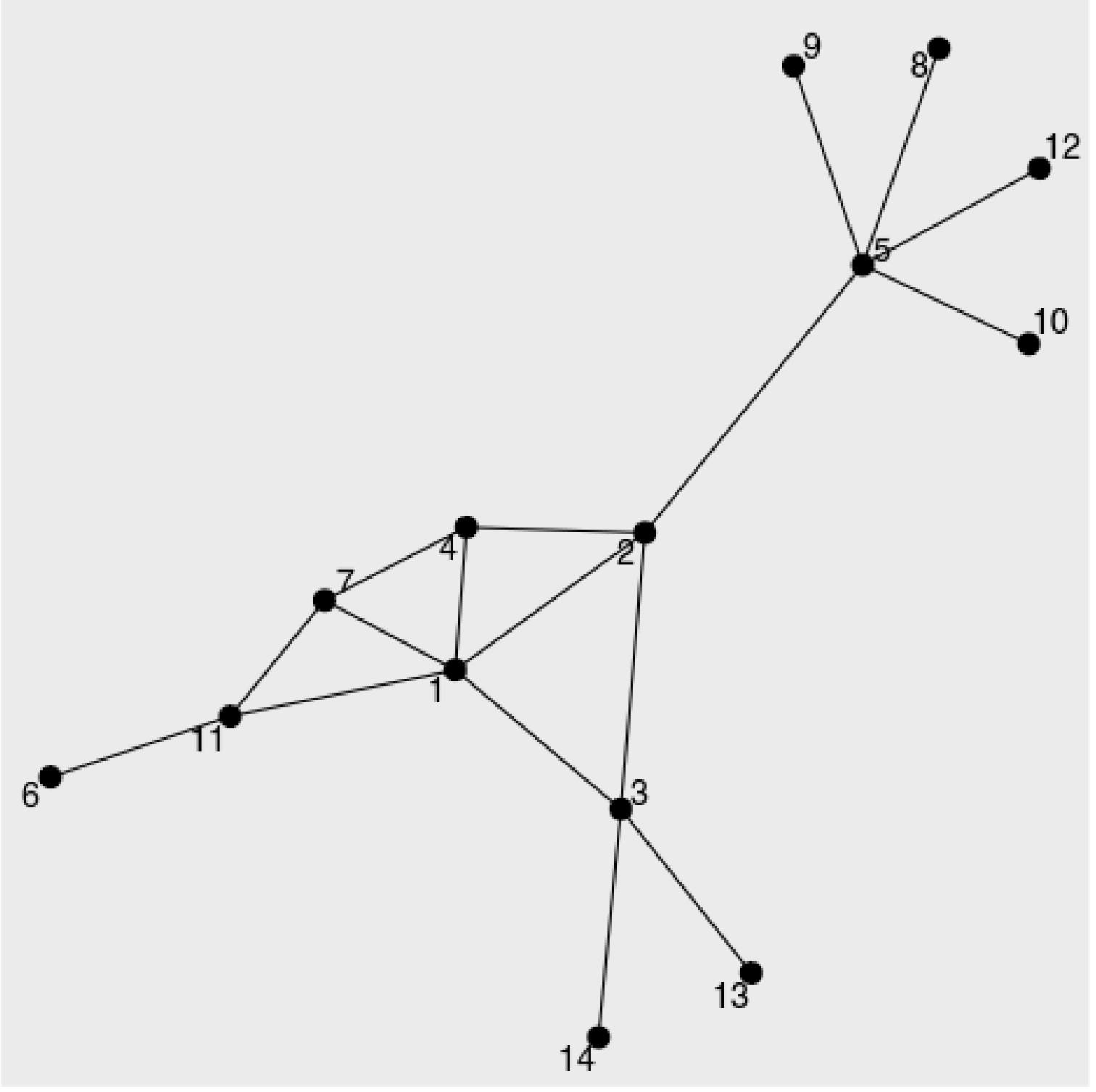

Figure 4. Fictitious social network for illustrating centrality ideas.

centrality measure. It essentially quantifies how often a vertex is found on the shortest path between two other vertices, and in figure 4 vertices 5 and 2 score highly.

Bonacich strove to address the effect of the surrounding environment of each vertex (see [14] and refs therein), with the idea that the importance of the vertices connected to the individual defines the importance of the individual. This idea was used to create a family of metrics that rate vertices highly if they are in turn connected to highlyrated vertices. The simplest of these is the eigenvector centrality that calculates the eigenvectors of the (zero diagonal) adjacency matrix. This is more useful than the plain degree centrality, and identifies vertex 1 and its surroundings as being the most important in figure 4 .

Bonacich then develops an expanded metric, which he calls $c(\alpha, \beta)$, where the $\beta$ 
parameter is used to describe the nature of the interaction that one wishes to quantify. Setting $\beta=0, c(\alpha, \beta)$ is proportional to simple degree centrality. Excursions into positivity, $\beta \rightarrow 1$, apply greater weight to the spread of message sending power to increasingly distant vertices.

Negative values, with $\beta \rightarrow-1$, place greater weight on a form of "dependency", where high-scoring vertices are surrounded by weak vertices. A high score with negative $\beta$ would indicate a person who acts as the sole information gateway (in a negative connotation) or as the key insulation from dysfunctionality (in a positive connotation) between clusters in the social network.

Borgatti [15] computes a metric in a more extreme way in a pair of "key player problem" metrics (KPP). The negative calculation, $\mathrm{KPP}-\mathrm{Neg}(k)$, involves computing the maximal fragmentation of the network on removing a subset of size $k$ of the vertices. This answers the question "Which $k$ nodes, if removed, would cause the most disruption?", and identifies important vertices in a more critical way than betweenness centrality. The choice of $k$ obviously has some bearing on the results. For example, it is possible for the vertex identified from the $k=1$ computation to not feature as part of the solution for some $k>1$. It is quite convenient to loop through a range of values of $k$, as Borgatti does. There is some correlation between a tally of Borgatti's KPP-Neg and Bonacich's $c(\alpha, \beta<0)$ but the two calculations are not equivalent.

$\operatorname{KPP}-\operatorname{Pos}(k)$, on the other hand, identifies set of vertices that maximally reach the greatest number of other vertices with the smallest number of steps. When planting $k$ copies of a message into a network, this metric identifies the most efficient nodes to disseminate information as widely as possible. Whereas a degree centrality calculation might naïvely suggest targeting the $k$ nodes with the most subscribers in an "influencer" marketing campaign, if the top 10 influencers all have the same subscriber and friend lists then KPP-Pos $(k)$ offers an opportunity to improve the propagation of information for the same amount of effort. The same arguments can be made for spreading misinformation through espionage or criminal networks.

KPP-Pos(1) identifies the communication centre of the network reliably. In an organisational structure, one would expect that KPP-Pos(1) identifies the CEO, and if it does not then one can take steps to correct this. In the example shown here, KPP$\operatorname{Pos}(1)$ identifies vertex 2 . Once again, there is a correlation between KPP-Pos and $c(\alpha, \beta>0)$, but the two are not equivalent.

The full set of metrics for the example graph from figure 4 are shown in table 1.2. Comparing the different columns, one sees:

- Vertex 1 is most central in positive adjacency metrics that typically measure authority and social prestige (degree, eigenvalue, $c(\alpha, \beta>0)$

- Vertex 5 is most central in communication-critical metrics (degree, betweenness, $c(\alpha, \beta<0, \mathrm{KPP}-\mathrm{Pos})$

- Vertices 2 and 3 also score highly in multiple areas, and would be worthy of further scrutiny 
Productivity Curve and Social Network Analysis in Science Megaproject Management 15

Table 2. Centrality metrics described in the text for the example network in figure 4

\begin{tabular}{rrrrrrrr}
\hline Vertex & Deg & Eigen & Btw & $c(\alpha, \beta>0)$ & $c(\alpha, \beta<0)$ & $\mathrm{kn} 10$ & $\mathrm{kp} 10$ \\
\hline $\mathbf{1}$ & $\mathbf{5 . 0 0}$ & $\mathbf{1 . 0 0}$ & $\mathbf{2 6 . 5 0}$ & $\mathbf{1 . 8 5}$ & $\mathbf{1 . 5 8}$ & $\mathbf{7 . 0 0}$ & $\mathbf{5 . 0 0}$ \\
2 & 4.00 & 0.81 & 41.50 & 1.59 & 0.79 & 3.00 & 5.00 \\
3 & 4.00 & 0.65 & 23.00 & 1.31 & 1.52 & 9.00 & 6.00 \\
4 & 3.00 & 0.73 & 3.00 & 1.27 & 0.91 & 3.00 & 2.00 \\
$\mathbf{5}$ & $\mathbf{5 . 0 0}$ & $\mathbf{0 . 3 7}$ & $\mathbf{4 2 . 0 0}$ & $\mathbf{1 . 1 8}$ & $\mathbf{2 . 4 1}$ & $\mathbf{1 0 . 0 0}$ & $\mathbf{9 . 0 0}$ \\
6 & 1.00 & 0.16 & 0.00 & 0.32 & 0.32 & 5.00 & 4.00 \\
7 & 3.00 & 0.67 & 1.00 & 1.18 & 0.86 & 5.00 & 2.00 \\
8 & 1.00 & 0.11 & 0.00 & 0.35 & 0.04 & 0.00 & 3.00 \\
9 & 1.00 & 0.11 & 0.00 & 0.35 & 0.04 & 3.00 & 4.00 \\
10 & 1.00 & 0.11 & 0.00 & 0.35 & 0.04 & 3.00 & 5.00 \\
11 & 3.00 & 0.54 & 12.00 & 1.02 & 1.01 & 4.00 & 3.00 \\
12 & 1.00 & 0.11 & 0.00 & 0.35 & 0.04 & 1.00 & 1.00 \\
13 & 1.00 & 0.19 & 0.00 & 0.38 & 0.22 & 1.00 & 3.00 \\
14 & 1.00 & 0.19 & 0.00 & 0.38 & 0.22 & 1.00 & 3.00 \\
\hline
\end{tabular}

- KPP-Neg and KPP-Pos tend to be somewhat correlated

It is interesting that the four vertices mentioned above are simply those with the largest degree centrality! This is not expected to be a general case. There are only 14 vertices in this graph, and in organisations of hundreds or thousands of people, or social networks with millions, it would be erroneous to assume that these conclusions can be extrapolated.

\subsection{Optimum Organisational Structure}

The final point to be introduced is the matter of optimum tree structure in the administration of a body of workers. The motivation for examining this point is that a recent review of a similar European project, namely the fusion project ITER [16]. That report identified that an entire layer of management could be removed, and in so doing there would be an improvement in efficiency and decision making.

One way of detecting managerial inefficiency is to simply look at the number of layers in the organisational structure. The absolute optimum number of layers below the CEO, $l_{m}$, ignoring specialist senior roles, and concentrating purely on administering delegation, is a well known equation in computer science coming from graph theory. The $m$-ary tree height (number of layers excluding the root, or CEO), depends on the team size, $s$, via the equation:

$$
l_{m}=\left\lfloor\log _{s}((s-1) \times n)\right\rfloor
$$

where $\lfloor x\rfloor$ is the floor function of $x$, and $n$ is the number of employees that must be managed (i.e. number of leaves on the tree structure). If one accepts a modern 
agile approach, then $s \sim 5-9$ is probably optimum [13]. As one increases the size of a team, the management costs per employee decrease. On the other hand, a larger team is unwieldy to manage: the number of communication channels within the team scales as $s(s-1) / 2$.

The present paper does not look at the authority balances between administrative and technical hierarchies and associated prestige, as famously discussed by Welchman [17] and Brooks [18]. Whilst these are important factors to optimise in any organisation, and can easily be added to the algorithms concerned, that problem is beyond the scope of what is intended here: the identification of a lower limit in the combined weight of management roles for such projects.

\section{Method}

\subsection{Long Tailed Distributions}

The data were analysed using the $\mathrm{R}$ language [19]. Each curve was analysed using either the "poweRlaw" package [20] or, if that analysis fails to support a power law then the fitdistrplus package was used [21]. poweRlaw uses maximum likelihood estimation (MLE), whilst fitdistrplus was used with numerical MLE or quantile matching estimation (QME) as appropriate. QME tends to drive the fit more towards the points in the tails of the distribution than the middle. The "Q-Q" plot (comparing empirical quantiles with the theoretical quantiles) is particularly useful in this regard.

In both cases, displaying the data and fits as a CCDF as used by Clauset et al [10] and Gillespie [20] (even though they call it "CDF") is used throughout this paper, since it illustrates the quality of the fit of the long tail much more usefully than the CDF method from fitdistrplus. It is also in keeping with the original work of Price, Lotka etc, whose data are presented in CCDF form.

\subsection{Social Network and Centrality}

The social network was analysed in two separate structures. The first is the plain, hierarchical structure as defined by management and HR. The organisation does, however, contain a multitude of committees, focus groups, task forces, and other cross functional teams that shall be henceforth known only as "committees". The most prominent four of these are the two committees charged to oversee health and safety, the executive committee, and a large body for reviewing analyses and change requests to approve technical decisions. This decision committee is the largest.

The $R$ package "igraph" [22] (along with the tidygraph API) was used for most of

the centrality measures. "influenceR" [23] provides KPP-Pos. KPP-Neg was created using the same greedy algorithm as described in Borgatti's paper [15]. 


\subsection{Optimum Organisational Structure}

Partitioning the workforce into a hierarchical structure of teams is not as simple as deciding the optimum team size and building a tree, one needs to consider grouping of similar roles and skill sets to create a harmonious and compatible mix in each department. The present article used a mix of three methods to achieve this. The first is a rules-based, sorted $m$-ary tree, written in base $\mathrm{R}$ [19] (a good introduction to trees and sorting is given by Sedgewick [24]). The second method was a genetic algorithm [25], and the third was particle swarm optimisation [26]; the latter two methods are types of metaheuristic algorithms that are useful for optimisation in non-linear problem spaces, and these were written in $\mathrm{C}++$. The sorted tree is a brute-force method that will find the global minimum given enough time, whilst metaheuristics tend to find good local optima in large problem spaces. For medium-sized organisations such as the one in this study, with $\sim 500$ employees, sorted $m$-ary trees converge in negligible time; in the general case including much larger organisations the metaheuristic methods will become increasingly applicable as the number of employees increases.

To visually map out the location of the high performance staff in the organisation, we need to be able to compare the performance of scientifically active staff with those producing collaborative support documentation. It is much easier to produce an administrative procedure or concept description than it is to write a peer-reviewed scientific publication. The administrative curve was normalised so that 6 pieces of administrative support roughly equal 1 scientific paper, which sets the most prolific administrative worker on equal footing with the most prolific scientist.

\section{Results}

\subsection{Performance Curves}

The research effort by the organisation falls almost exactly on the expected log-normal curve. Indeed, the solid line fit of the data from Price's paper provide a more satisfactory description of the curve than a direct fit of the data, due to some statistical fluctuations (or systematic effects) in the middle of the curves.

For some time, the most puzzling aspect of the analysis was in the administrative performance curve. Here, it was not possible to find a single distribution function to describe all the data, instead there appears to be a piece-wise power law behaviour, where the top portion of the $c c d f()$-transformed data is consistent with a square root power law, and the highest performance staff follow a second power law exponent, with a clear demarcation between the two groups. It seemed immediately plausible that these data indicate two distinct social groups, one "clique" that works in one environment, and "outsiders" that experience another working environment, distinguished by these exponents, but it was not easy to find any literature on such a mixed system.

It is possible to reproduce this behaviour with a Monte-Carlo model. The clique is reproduced by a log-normal tail of multi-trial challenges. Outside the clique, it doesn't 


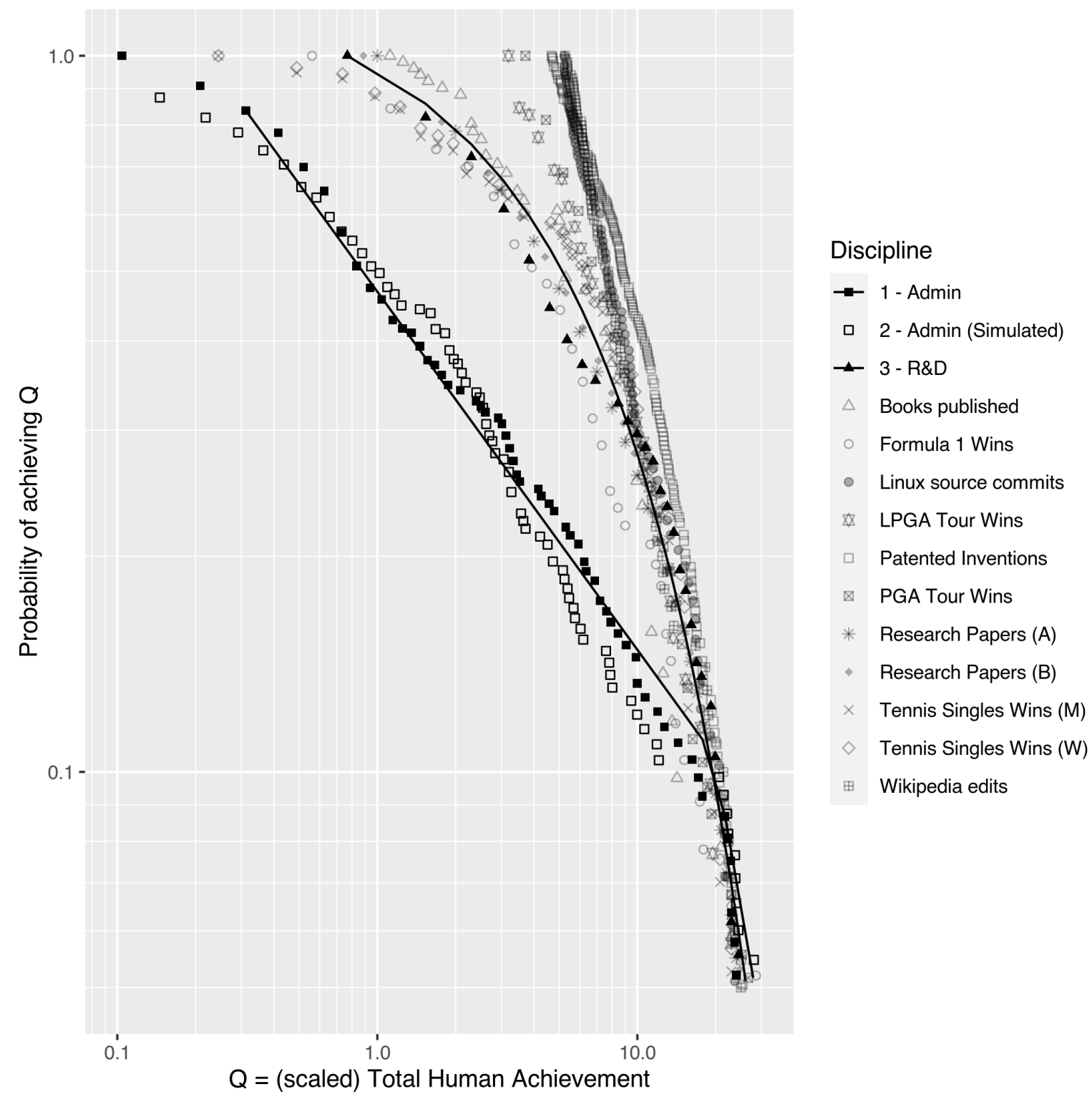

Figure 5. Measured admin performance compared to a "clique" and "outsider" Monte-Carlo model, where the "outsiders" face additional hurdles in the completion of work units compared to the "clique", yielding productivity curves resembling a piecewise power law. The grey curves are the same data from figure 1 that have been shifted to show the almost universal relative behaviour in each field of endeavour.

matter whether the challenges are repetitive uses of a single skill or draw on multiple skills. The number of challenges needed to be overcome in order to successfully complete work are simply doubled compared to those within the clique. This produces a piece-wise productivity curve with an approximate square-root law over two orders of magnitude of work output and a log-normal tail, as shown in figure 5 .

The clique-and-outsider behaviour could have many origins. One explanation is that the clique have ingratiated themselves with management, and therefore have easy access to an express approval channel by their close contact with authority figures, 
which is certainly supported by the graph analysis. Another explanation would be managers rejecting work more readily if it comes from non-favoured staff, requiring more confirmation data in order to be persuaded. This is not necessarily outright cronyism - it could equally arise with managers being more likely to accept completed work that they themselves have initiated with trusted subordinates, whilst voicing scepticism at new initiatives that originate from those outside their usual collaborative circles - a form of creativity bias [27]. Another alternative is a stratification into a minority of enfranchised staff and a majority of disenfranchised staff (if driven by a topdown mechanism) or a minority of "engaged" staff and a majority of those who have "disengaged", in a more bottom-up scenario. All of these are plausible in a decade long project, and one finds anecdotes consistent with all of these mechanisms, to varying degrees, in many organisations.

The end result is poor social cohesion in the workplace and a suppression of productivity. The penalty appears to be $50 \%$ in total work output, interpreting the central, empty region separating the admin curve from all the other types of curve as missing work. If Price's law had held for this data, we would anticipate that of 135 active in the administration work that half of all the work was done by $\sim 12$ people. However, because of the reduced productivity in the outsiders' group, half of all the administrative work is done by just four people.

If the situation could be somehow improved, then the outsider data would shift to the right and lie near the straight line behaviour over a greater portion of the workforce. It represents almost doubling the work output across a large fraction of the organisation.

As mentioned earlier in this article, there are other systems that could also be data mined (e.g. engineering collaboration tools) and one might also imagine that this square-root behaviour could be generated by a large fraction of the workforce spending roughly half their time in the other systems that are out of the scope of the present analysis. Whilst that is a mathematically equivalent scenario, this was ruled out for two reasons. Firstly, there are a large number of staff who appear on both the research and administration productivity curves: their research work output does not exhibit any strong, piecewise anomaly as seen in their administration work. Secondly, many of the "clique" group are known to be prolific users of the other systems also.

\subsection{Organisational Structure}

The social structure of the organisation will now be examined, starting with the baseline organisation from 2018.

\subsection{Baseline}

The baseline organisational network is shown in figure 6 both with plain hierarchy and and with committees.

Excluding the committee structure, the three most crucial nodes, via $\operatorname{kppneg}(3)$, identify the following staff as retention priorities: 

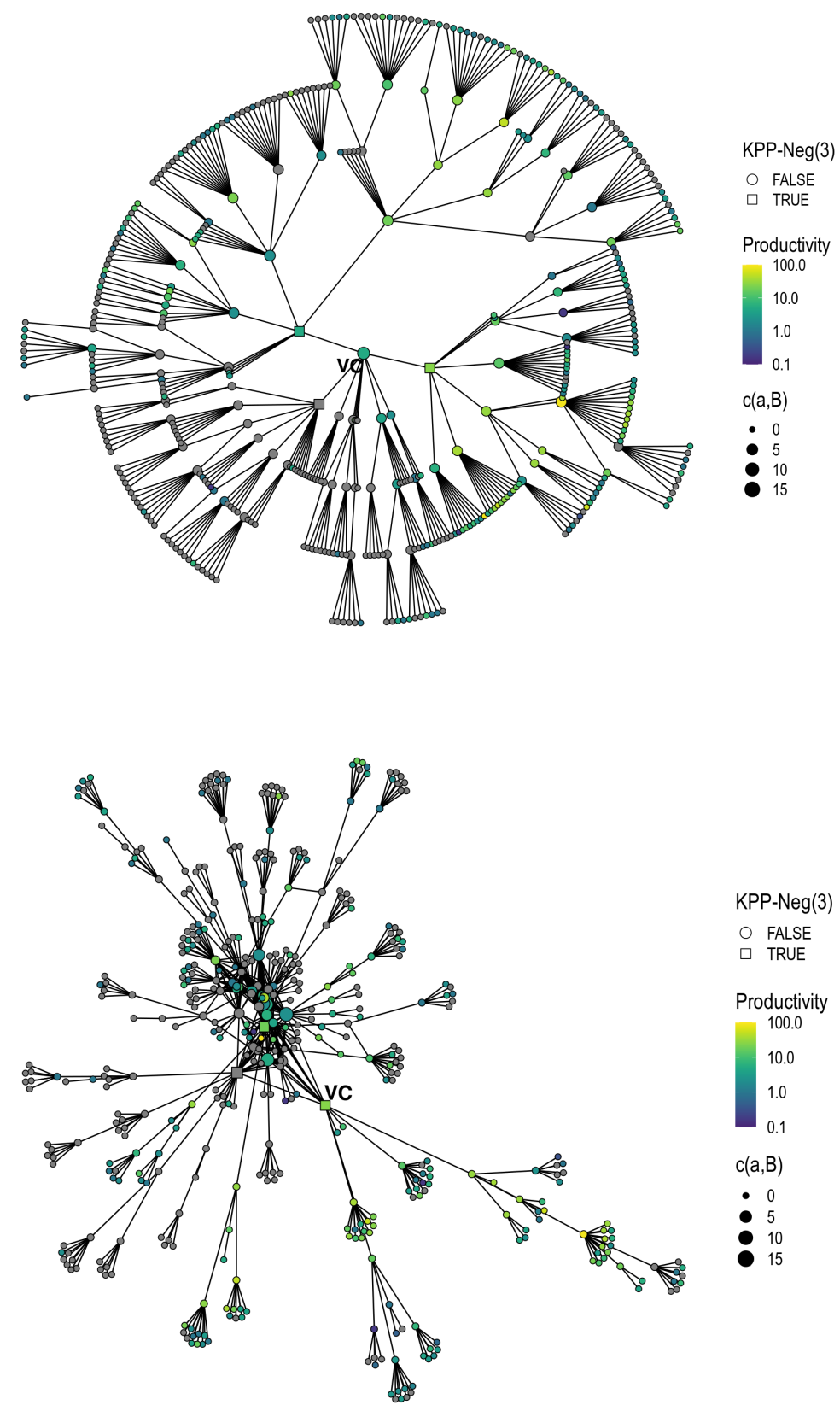

Figure 6. Baseline organisational structure showing plain hierarchy (top) and including social interactions from committee membership (bottom). The node marked "VC" indicates the critical node as described in the text; squares indicate retention priorities; the colour scale indicates the productivity of the worker in support of research department activities on a log scale; and the symbol size indicates centrality measure $\mathrm{c}(\alpha, \beta>1)$. 
- Technical director

- Director of the research department

- Director of the administration department

The single most critical retention, via kppneg(1), is marked "VC" and is the CEO. With committee networks included, the three most crucial nodes, via kppneg(3), identify the following staff as retention priorities:

- Director of the administration department

- Technical division head (not the technical director, but their subordinate)

- Director of the research department

The single most critical retention, via kppneg(1), is the director of the research department, and not the CEO. There could be two reasons for this: either delegation of responsibilities - which would be a positive explanation - or a more remote leadership figure than one would like, which is of course more negative. Which of these is taking place here is beyond the scope of this project, and could be uncovered fairly easily with further investigation.

When looking at the network structure as a whole, the first observation is that there is very little matrix activity linking the expert teams further out with the decision making committees at the centre. Instead, the committees are densely bundled, recyling the same sets of senior managers, and the expert teams are only connected into the decision making processes via formal hierarchy. This offers an immediate insight into how some stratification in working culture might emerge, as seen in figure 5.

Also, one can see that the majority of high performing individuals are located outside the dense cluster of committees, and that productive staff tend to be somewhat clustered. Only one senior manager is present in the central committee cluster and the top 20 performing staff. What was interesting about this manager in particular was their public declarations on open working practices, enshrined in the core values of openness and transparency, whilst at the same time enforcing a culture of secrecy in their department. After some further investigation, it was found that secrecy was stated as an actively chosen strategy for half of the highest performing staff in research, to avoid the impact that interference had on their work. To balance the contradiction, they also described a culture of selective sharing, where some moderately important work was shared to give the impression of proactive openness to those senior in the hierarchy, whilst at the same time hiding the most important work from view for as long as possible. In this context, it is interesting that the most strongly correlated centrality measure with research performance is $c(\alpha, \beta>1)$, which measures the ability of a vertex to communicate widely. Whilst the correlation is relatively mild (Pearson correlation coefficient $\rho \approx 0.5$ ) it restores faith in the idea that openness and collaboration are beneficial to research work, even in an organisation where many feel it necessary to behave secretively. 


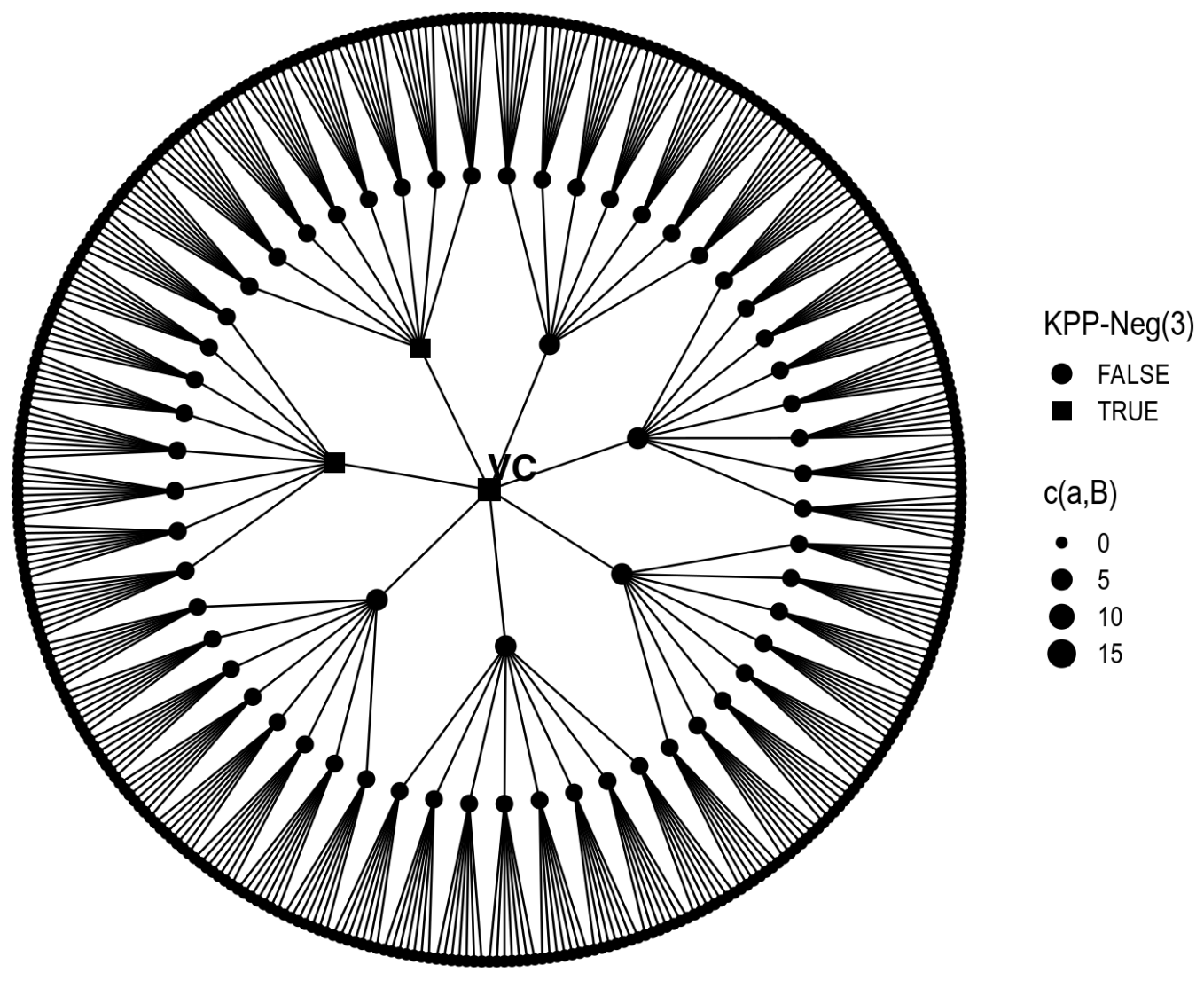

Figure 7. Proposed organisational structure showing plain hierarchy. The node marked "VC" indicates the critical node as described in the text; squares indicate retention priorities; and the symbol size indicates centrality measure $\mathrm{c}(\alpha, \beta>1)$.

\subsection{Proposed Structure}

The proposed organisational structure, generated as described in section 2.3, is shown in figure 7 in plain hierarchy form.

The CEO is still the critical retention with kppneg(1), however this structure reduces the annual salary budget by $10 \%$ by eliminating two layers of superfluous, middle management roles. The number of managers has decreased from 95 to 60 , of which the number of directors is reduced from 11 to 7 . The cost savings are approximately $€ 3 \mathrm{M}$ per year, based on rough estimates and the publicly-shared standard costs for each role.

One might at this stage question whether the middle management roles are 
productive or valuable, and if eliminating them poses some risk. Certainly inspection of figure 6 indicates that neither of the two quantified outputs are correlated with managerial rank. An important point to bear in mind, with a top-heavy organisation of this kind, has been described by Graeber [28]. Expanding middle management undoubtedly expands, proportionally, the work that must be done to satisfy the needs expressed by those in these positions. This might take the form of additional paperwork, requests for explanations, reviews, or meetings; new committees and task forces to scrutinise key areas. Some of these will no doubt be useful. Indeed, in all of the international reviews that were held, technical risks were highlighted and result in recruitment to mitigate in crucial and often overlooked areas, to avoid pitfalls from prior experience. Examples include systems engineering, fire safety, earthquake safety, IT infrastructure, data and software, and cybersecurity. A handful of senior managers cannot possibly possess the required expertise in all areas to answer to these risks.

There is a temptation for management to satisfy the board of their credibility by recruiting positions to handle these risks at prominent, visible levels. Despite the usefulness of many of these roles, there are undoubtedly some that create potent distractions. Furthermore, it is a mathematical feature of the tree structure that there are many more subject matter experts in the lower levels with $\mathrm{PhDs}$, masters degrees, and several years of fresh, relevant, engineering and technical experience that would provide greater value for money and efficiency if their expertise were empowered to the same degree.

To understand which of the middle management roles could be eliminated, one can consider the categories of dysfunctional roles that Graeber identifies and modify them in the context of science megaprojects:

Flunkies who are accumulated by their superiors to create visible demonstrations of their importance or credibility

Goons who provide muscle, manipulation, deception, or act as "independent reviewers/approvers"

Duct-tapers who are tasked to provide "quick fix" services instead of permanent solutions

Box-tickers who accumulate administrative work as an exercise unto itself

Taskmasters who manage and create extra work

The objective is to identify the branches of management where dysfunction exists, amongst functional management practice. Fortunately, spotting these low-value roles, and more importantly identifying the senior figures who create them, is a fairly easy exercise.

Very often, flunkies will be searching for useful tasks to perform, beyond their allocated tasks of standing in their manager's shadow, taking notes in meetings, and forwarding correspondence on their behalf. Senior flunkies usually have vague, impressive-sounding roles and few or no subordinates. They busy themselves by attending committee meetings and/or acting as taskmasters. 
Goons may include those who are formally project "sponsors" who, instead of fulfilling the role of sponsor [29], micromanage all aspects of the project at the request of a senior figure. The approver goon is highly visible: these positions frequently cycle through expert after expert whilst management searches for someone who is prepared to support their position, rather than asking the experts to create a bottom-up plan of their own. In the long term, it is likely to be filled by someone prominently lacking in qualifications for the area in which they are signing. The potential damage created by this role type is perhaps clearer when one considers instead of "goon" the terminology of a "henchman" who supports/instigates workplace bullying [30] at the behest of more senior figures.

Duct-tapers are often dissatisfied professionals who complain that they are being given detailed instructions on downgrading their work below best practice in order to reduce costs. In science, they are sometimes, quite literally, duct tapers, rushing to fix vacuum seals with tape because (a) the decision was made to use flimsy, cheap solutions instead of robust engineering and best practice; and (b) another decision was made that an extended maintenance shutdown to replace defective components is unacceptable. The famous saying "buy cheap, buy twice" is often at play. The irony is that the salaried time of the duct tapers usually exceeds the replacement cost, and even more so the marginal cost of the best practice solution in the first place.

Box-tickers frequently appear at interfaces between technical areas and departments in projects that are heavy on paperwork, contract-style agreements, secrecy, and waterfall planning - in other words, the polar opposite of the agile manifesto. However, it is not the box-tickers themselves who are the unnecessary cost, often they are highly knowledgeable engineers, but it is the staff who task the box-tickers: usually the taskmasters and their superiors.

Some of this dysfunctionality can be found via a mathematical inspection of the organisational structure, without deep social investigation. Figure 8 shows the same organisation, without committee interactions, as given in figure 6. In this case, however, the figure is labelled so that one can identify potential dysfunctionalities in the social arrangement of each node. The symbols each indicate the possibility of:

++ leading a team that is too large

- - leading a team that is too small

GC occupying a senior management role with no subordinates

MM being a member of a team that is too large or too small

ND being a senior manager who recruits their own staff instead of delegating (often duplicating roles and responsibilities existing elsewhere)

SR being a manager whose job it is to manage a single person

None being in an optimum organisational situation

"++", "ND", and those to whom "GC" report, are consistent with managers deserving some investigation into their operations, particularly "ND". 


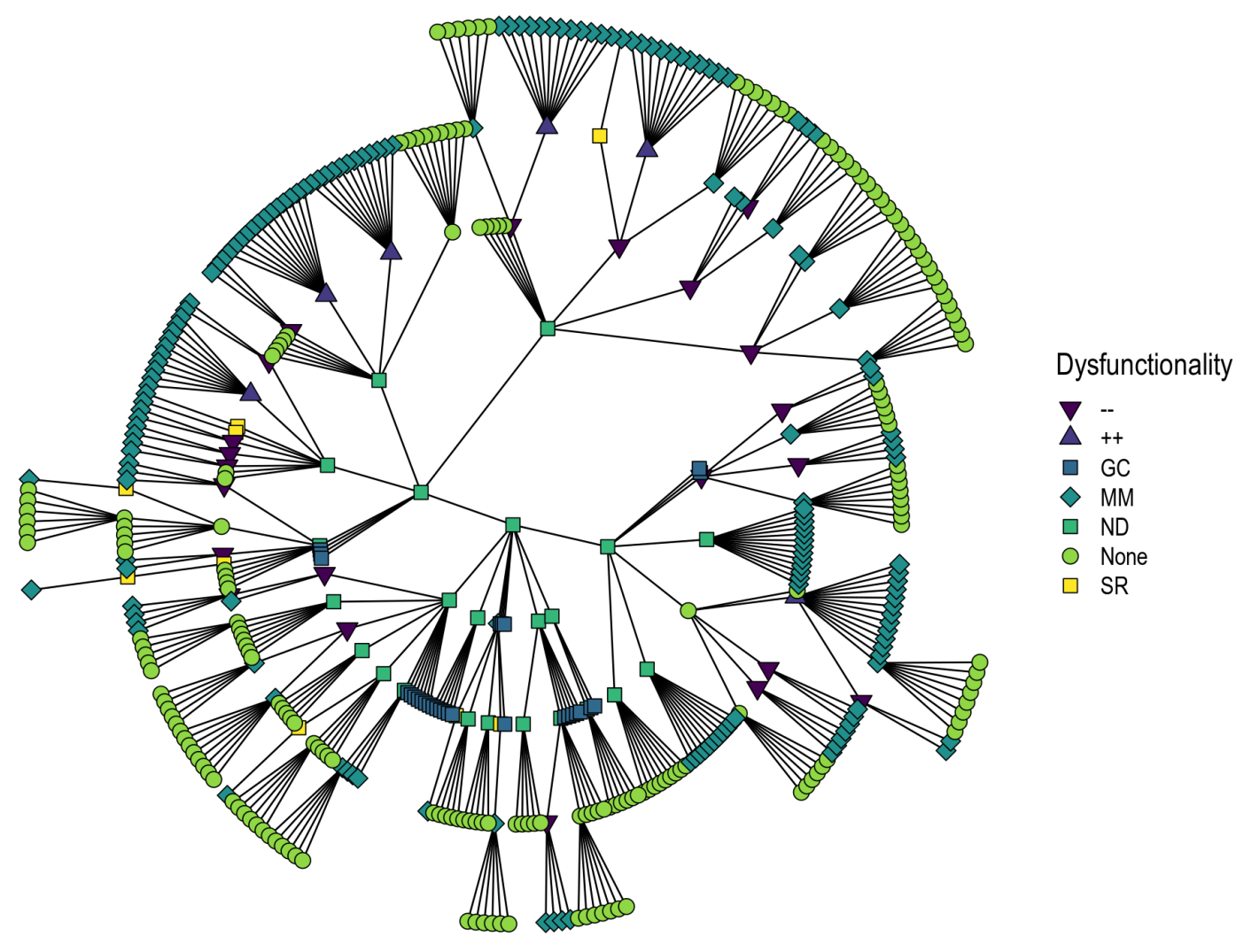

Figure 8. Baseline organisational structure showing plain hierarchy, the same arrangement as for figure 6 except this time the colour and symbol indicate a potential dysfunctionality in the social structure around the vertex as indicated in the legend and described in the text.

We see in figure 8 that 253 employees out of 455 are possibly subject to an organisational dysfunctionality, representing $56 \%$ of the workforce. In contrast, the software that generates figure 7 is designed to eliminate all of those effects described above, leading to $0 \%$ organisational dysfunctionalities. Of course, this does not guarantee zero problems, but it offers a starting point from which a pragmatic structure could be built.

\subsection{Management's Chosen Structure}

The analysis in the previous section was presented to management at the beginning of 2019. Afterwards, management undertook their own reorganisation work, and their structure is shown in figure 9, in plain hierarchy and committee form. Locally there are some changes, but globally it is almost identical to the baseline. As a result, the restructuring work cannot be expected to fix any of the underlying problems facing the organisation, and in fact may have caused further deterioration in some areas. 

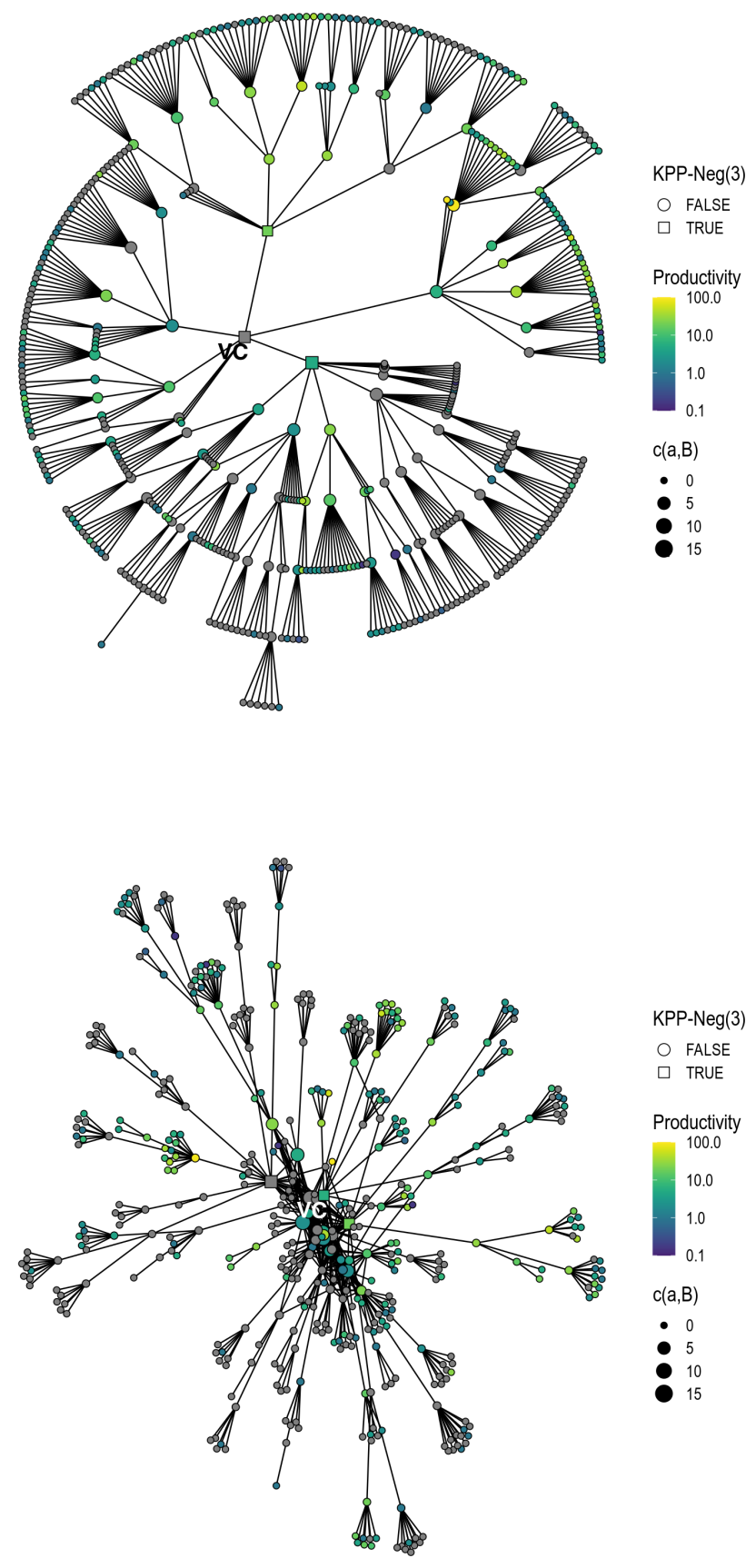

Figure 9. Management's chosen reorganisation structure showing plain hierarchy (top); including social interactions from committee membership (bottom). The node marked "VC" indicates the critical node as described in the text, whilst squares indicate possible retention priorities and the colour scale indicates the productivity of the worker in support of research department activities on a log scale; and the symbol size indicates centrality measure $\mathrm{c}(\alpha, \beta>1)$. 
Excluding the committee structure, the three crucial nodes via kppneg(3) are:

- Project director

- Technical division head (not the technical director, but their subordinate)

- The CEO

The critical retention kppneg(1) is again not the CEO, but the project director. Including the committee structure, the three most crucial nodes, once more identified via kppneg(3), are:

- Director of the administration department (as in baseline)

- Technical division head (not the director, as in baseline)

- A mid-level project manager

The single most critical retention, identified with kppneg(1), is the same mid-level project manager as identified with kppneg(3).

It is interesting that the director of research has become less critical in the new structure, and essentially replaced by a mid-level project manager. The project manager is prominent in the baseline structure of figure 8 for being quite high in their "ND" dysfunctionality score. One can be quite confident that neither the CEO nor the director of research would intend for this to have happened by design.

Figure 10 shows the chosen management structure once more, highlighting potential dysfunctionality of various types as done before for the baseline in figure 8. The differences between figure 8 and 10 are as follows:

- The number of senior staff with no direct subordinates has expanded from 29 positions to 38

- The number of managers possibly not delegating functionally remains static at 23 .

- The number of supervising managers has decreased by 1

- The number of "coalface" workers (those working more than 2 steps away from the CEO with no direct subordinates) has increased by 9 .

Thus we see a slight overall expansion of the workforce by $9+9-1=17$ positions, and more than half of those are in senior organisational roles with no supervisory responsibilities.

\section{Discussion and Conclusions}

The productivity curves and social network of a European science megaproject have been analysed. Whilst the research output matches the expected behaviour, the administrative work in support of project activities and the social network analysis reveal several aspects of a core problem:

- A stratified working culture

- A small clique with high administrative productivity 


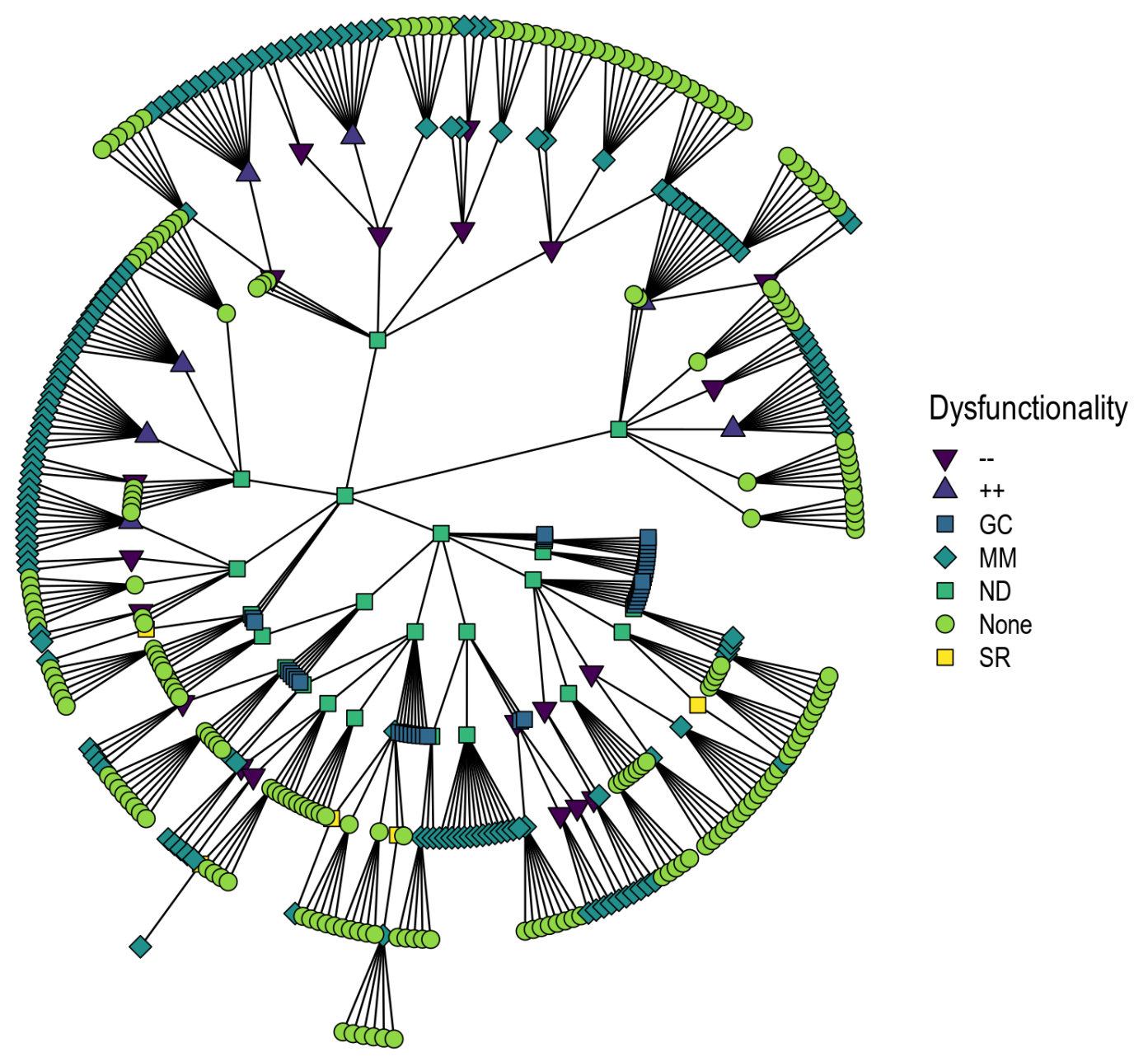

Figure 10. Management's chosen organisational structure showing plain hierarchy, the same arrangement as for figure 9 except this time the colour and symbol indicate a potential dysfunctionality in the social structure around the vertex as indicated in the legend and described in the text.

- An outsider group whose productivity is significantly reduced to around $50 \%$ of the potential output

- A dense network of decision makers who are connected to the expert teams via hierarchy

- A culture of secrecy in the more productive research staff

- More than half of the workforce in a non-optimal social structure

- An excess of management roles, many having no subordinates and many conversely recruiting their own teams instead of delegating to existing expert groups

These effects seem to be disconnected but in fact share a likely source, which is 
the excessive layers of management. The same conclusion was drawn in a review of a similar scientific megaproject [16], and the way it arises is rational and understandable. The interventions of the break-fix model, both in the form of regular project reviews and corrective re-planning, create prominent senior recruitments in order to improve credibility and trust.

Unfortunately, the suppression of productivity associated with excessive management is well grounded: recent estimates show that each layer of management adds roughly $10 \%$ to the administrative workload of an employee, and successful companies have between 3 and 4 layers [31, 32] — precisely as shown in figure 7 .

It is interesting that the stratification is not visible in the research productivity curve. One possible explanation for this is that scientific interest and research is very much a vocation for those who pursue it, whilst project work is "part of the job". When the "job" environment breaks down due to the megaproject issues mentioned before, then those with the vocational attitudes immerse themselves in their academic interests. As a contributing mechanism, one should not rule out the link between the goon/henchman dysfunctional management roles and the degree to which bullying behaviour spreads outside the immediate instigator-victim circle [].

Doubling the work output of a large fraction of the workforce carries a cashequivalent benefit that greatly exceeds anything achievable via a more traditional topdown optimisation of available effort, such as replanning and reassigning work, moving teams between departments, or increasing the number of facilitators and coordinators. The pressing concern for megaproject efficiency, therefore, is to remove the superfluous managers that score highly in dysfunctional environment metrics and/or possess obvious dysfunctional roles defined in section 3.4. Instead, one should directly engage the expert teams in the decision making processes and committees and nurture more bottom-up approaches.

To improve any residual disengagement, cronyism, negative creativity bias, or favouritism, will probably require additional steps that are outside the scope of this paper: a recent literature review identified 18 problems and 54 "cures" documented in academic publications on megaprojects [33]. Corrective action is likely to include a series of measures to improve the understanding of roles [29]; increase project ownership, delegation, and empowerment (e.g. [34]); increase collaboration around shared technical goals (e.g. [13]); and to reduce risk/creativity aversion associated with the uncertainty that comes with breaking new technical ground [27].

There appears to be a disproportionate, beneficial impact available for the project in this study if this stratified culture could be corrected. Indeed, avoiding it entirely should be a priority for future science megaprojects. The estimates earlier in the article suggest that this would avoid annual financial losses of more than $10 \%$ of the annual salary budget ( $\sim 3 \mathrm{M} €$ per year), but that is the least significant result. The primary outcome of interest is unlocking the remaining 50\% productivity for the "outsiders" in the technical administration areas who could be defining, coordinating, and making decisions in their areas of expertise. A workforce operating at half its capacity is 
naturally expected to take twice as long to deliver planned units of work. Schedule slip is directly translated into cost overrun, estimated for this project to be $10-20 \mathrm{M} €$ per month, similar in proportion to other well-documented projects [3]. Avoiding such losses enables a facility to avoid commensurate reductions in scope and ambition of the facility, and to deliver the full science benefits that are anticipated when the project is first approved.

[1] P. M. Bentley. Instrument suite cost optimisation in a science megaproject. J. Phys. Commun., 4:045014, 2020.

[2] P. M. Bentley and U. Filges. Metaheuristic layout design of a 2 billion euro science facility. Journal of Physics Cummunications, 4(5):055011, 2020.

[3] B. Flyvbjerg. What you should know about megaprojects, and why: An overview. Project Management Journal, 45(2):6-19, 2014.

[4] B Flyvbjerg, editor. The Oxford Handbook of Megaproject Management. Oxford University Press, 2017.

[5] P. M. Bentley. Accurate simulation of neutrons in less than one minute pt. 2: Sandman - gpuaccelerated adjoint monte-carlo sampled acceptance diagrams. Quantum Beam Science, 4(2):24, 2020 .

[6] J. Welch and J. A. Byrne. Jack: Straight from the Gut. Grand Central Publishing, 2003.

[7] S. H. Moon, S. E. Scullen, and G. P. Latham. Precarious curve ahead: The effects of forced distribution rating systems on job performance. Human Resource Management Review, 26:166179, 2016.

[8] A. J. Lotka. The frequency distribution of scientific productivity. Journal of the Washington Academy of Sciences, 1926.

[9] D. J. de Soller Price. Little Science, Big Science... and beyond. Columbia University Press, NY, 1963, 1986.

[10] A. Clauset, C. Rohilla Shalizi, and M. E. J. Newman. Power-law distributions in empirical data. SIAM Review, 51(4):661-703, 2009.

[11] C. Kleiber and S. Kotz. Statistical Size Distributions in Economics and Actuarial Sciences. John Wiley \& Sons, Inc, 2003.

[12] A. Georgakopoulos, L. Wilkin, and B. Kent. Workplace bullying: A complex problem in contemporary organizations. International Journal of Business and Social Science, 2(3), 2011.

[13] K. S. Rubin. Essential Scrum. Addison Wesley, 2013.

[14] P. Bonacich. Power and centrality: A family of measures. American Journal of Sociology, 92(5):1170-1182, 1987.

[15] S. P. Borgatti. Identifying sets of key players in a social network. Comput Math Organiz Theor, 12:21-34, 2006.

[16] D. Clery. New review slams fusion project's management. Science, 343:957-958, 2014.

[17] G. Welchman. The Hut Six Story. Penguin, 1984.

[18] F. Brooks. The Mythical Man-Month: Essays on Software Engineering. Addison-Wesley, 1975.

[19] R Core Team. R: A Language and Environment for Statistical Computing. R Foundation for Statistical Computing, Vienna, Austria, 2020.

[20] C. S. Gillespie. Fitting heavy tailed distributions: The poweRlaw package. Journal of Statistical Software, 64(2):1-16, 2015.

[21] M. L. Delignette-Muller and C. Dutang. fitdistrplus: An R package for fitting distributions. Journal of Statistical Software, 64(4):1-34, 2015.

[22] G. Csardi and T. Nepusz. The igraph software package for complex network research. InterJournal, Complex Systems:1695, 2006.

[23] S. D. Jacobs and A. S. Khanna. influenceR: Software tools to quantify structural importance of nodes in a network. cran.r-project.org/package=influenceR, 2015.

[24] R. Sedgewick. Algorithms in C++. Addison-Wesley, 1998. 
[25] J. T. Holland. Adaptation in Natural and Artificial Systems. The MIT Press, 1975 \& 1992.

[26] J. Kennedy and R. Eberhart. Particle swarm optimisation. Proc. IEEE Int. Conf. Neural Netw., 4:1942-1948, 1995.

[27] J. S. Mueller, S. Melwani, and J. A. Goncalo. The bias against creativity: Why people desire but reject creative ideas. Psychological Science, 23(1):13-17, 2012.

[28] D. Graeber. Bullshit Jobs: A Theory. Simon \& Schuster, 2018.

[29] A Guide to the Project Management Body of Knowledge. Project Management Institute, 2013.

[30] P. Lutgen-Sandvik and V. McDermott. The constitution of employee-abusive organizations: A communication flows theory. Communication Theory, 18:304-333, 2008.

[31] Bartleby. The secret to cutting corporate red tape. The Economist, $13^{\text {th }}$ March 2021.

[32] M. Lindstrom. The Ministry of Common Sense: How to Eliminate Bureaucratic Red Tape, Bad Excuses, and Corporate BS. HMH Books, 2021.

[33] J. Denicol, A. Davies, and I. Krystallis. What are the causes and cures of poor megaproject performance? a systematic literature review and research agenda. Project Management Journal, 51(3):328-345, 2020.

[34] I. Fraser, K. Korterud, M. R. Wood, and C. Curran-Morton. Establishing benefits ownership and accountability. Project Management Institute - Thought Leadership Series, 2016. 